\title{
Passively mode-locked ytterbium-doped fiber laser with cylindrical vector beam generation based on mode selective coupler
}

\author{
Hongdan Wan, Jie Wang, Zuxing Zhang, Jinzhang Wang, Shuangchen Ruan and Lin Zhang
}

\begin{abstract}
We propose and demonstrate, for the first time to the best of our knowledge, a passively mode-locked ytterbium-doped fiber laser generating cylindrical vector beams (CVBs) using a mode selective coupler (MSC) as the transverse mode converter and splitter, and a semiconductor saturable absorber mirror (SESAM) for mode-locking. According to the coupling mode theory and the phase matching principle, the MSC was fabricated by weakly fused technology, having a low loss of about $0.5 \mathrm{~dB}$ and achieving LP11 mode with a high purity of $>96 \%$. The CVB fiber laser operates at a center wavelength of $1042.3 \mathrm{~nm}$, with a $3 \mathrm{~dB}$ spectral width of $1.5 \mathrm{~nm}$. The repetition rate of the mode-locked laser pulses is $18.58 \mathrm{MHz}$. The radially and azimuthally polarized vector beams can be switched by adjusting the polarization controllers in the fiber ring cavity, with a high mode purity measured to be $>\mathbf{9 5 \%}$. The mode-locked CVB ytterbium-doped fiber laser has potential applications in optical tweezers, optical imaging, and so on.
\end{abstract}

Index Terms-Cylindrical vector beam, laser mode locking, mode selective coupler.

\section{INTRODUCTION}

$\mathrm{T}$ he cylindrical vector beams (CVBs) including radially and azimuthally polarized beams have drawn considerable attention recently due to their unique axial symmetry in both field amplitude and polarization [1]. This polarization

Manuscript received xxxx. This work was supported by National Science Foundation of Jiangsu Province under (BK20150858, BK20161521), the Distinguished Professor Project of Jiangsu (RK002STP14001), the Talents Projects in Nanjing University of Posts and Telecommunications (NY214002, NY215002) and the STITP of NUPT (XZD2017040). (Corresponding authors: Hongdan Wan and Zuxing Zhang).

Hongdan Wan is with the Advanced Photonic Technology Lab, Nanjing University of Posts and Telecommunications, Nanjing 210023, China (e-mail: hdwan@njupt.edu.cn).

Zuxing Zhang is with the Advanced Photonic Technology Lab, Nanjing University of Posts and Telecommunications, Nanjing 210023, China and also with Aston Institute of Photonic Technologies, Aston University, Birmingham B4 7ET, UK (e-mail: zxzhang@njupt.edu.cn).

Jie Wang is with the Advanced Photonic Technology Lab, Nanjing University of Posts and Telecommunications, Nanjing 210023, China.

Jinzhang Wang, Shuangchen Ruan are with Shenzhen Key Laboratory of Laser Engineering, College of Optoelectronic Engineering, Shenzhen University, Shenzhen 518060, China (e-mail: jzwang@szu.edu.cn and scruan@szu.edu.cn).

Lin Zhang is with the Advanced Photonic Technology Lab, Nanjing University of Posts and Telecommunications, Nanjing 210023, China and also with Aston Institute of Photonic Technologies, Aston University, Birmingham B4 7ET, UK (e-mail: 1.zhang@aston.ac.uk). symmetry leads to interesting properties under high-NA focusing conditions [2].The CVBs have been widely used in particle physics [3], optical tweezers [4], material processing [5], high-resolution metrology [6] and surface plasmon excitation [7].

Various techniques have been proposed and demonstrated to generate CVBs, including the use of spatial polarization selective elements and the all-fiber methods. The spatial polarization selective elements including dual conical prism [8], birefringent components [9], spatial-light modulator (SLM) [10], and sub-wavelength gratings [11] were introduced into the laser cavity to generate CVBs. Comparatively, the all-fiber method has the advantages of lower loss, higher compactness and efficiency [12], [13], and can also effectively be combined with fiber laser's intracavity mode-locking and Q-switching techniques to generate pulsed CVBs. However, the all-fiber method usually involves offset splicing technique to excite high-order modes and few-mode fiber Bragg gratings (FM-FBGs) as the transverse-mode selector to achieve CVB generation [14]-[17]. Unfortunately, lateral misalignment between single mode fiber (SMF) and few-mode fiber (FMF) introduces relatively large insertion loss into laser cavity and deteriorates laser slope efficiency [18]. In order to improve laser performance, mode selective coupler (MSC) has been utilized to generate the CVB, which acts as both the mode converter and splitter. In Reference [19], we demonstrated a passively mode-locked Erbium-doped fiber laser generating pulsed CVBs based on an all-fiber MSC and nonlinear amplifying loop mirror (NALM). Recently, CVBs' generation based on MSCs is limited to Erbium-doped fiber lasers operating at the wavelength band of $1550 \mathrm{~nm}$ [18]-[20]. More efforts are still needed to extend the operating wavelength of CVB fiber laser to $1.0 \mu \mathrm{m}$ and develop high efficiency pulsed CVB fiber laser with a high purity.

In this paper, we firstly theoretically and experimentally present an all-fiber MSC capable of exciting LP11 mode in two mode fiber (TMF) with high mode purity and low loss at 1.0 $\mu \mathrm{m}$ wavelength band. Then, we propose and demonstrate an all-fiber pulsed ytterbium-doped CVB fiber laser based on the fabricated MSC. To the best of our knowledge, this is the first report on pulsed CVB ytterbium-doped fiber laser with high efficiency and mode purity operating at wavelength band of 1.0 $\mu \mathrm{m}$, using an all-fiber MSC for mode conversion and a SESAM for mode-locking. 


\section{Theoretical ANALYSIS AND EXPERIMENTAL SETUP}



Fig. 1. Schematic of the MSC. The LP01 mode is launched into the SMF input port, the LP11 mode is expected to be excited at the TMF output port, while the uncoupled LP01 mode will propagate along the SMF.

The basic structure of the MSC is depicted in Fig. 1. It consists of a TMF and a SMF (core/cladding diameter = $6.2 / 125 \mu \mathrm{m}, \mathrm{NA}=0.14)$. The conventional single mode telecom fiber (SMF-28, core/cladding diameter $=8.2 / 125 \mu \mathrm{m}$, $\mathrm{NA}=0.14)$ is used as the TMF at the wavelength of $1.0 \mu \mathrm{m}$. The operation principle of the MSC is to phase match the fundamental mode in the SMF with a high-order mode in the TMF, and achieve mode conversion from fundamental mode to high-order mode [21].

According to the coupling mode equations [22]:

$$
\begin{aligned}
& P_{1}(z)=\left|A_{1}(z)\right|^{2}=1-F^{2} \sin ^{2}\left(\frac{C}{F} z\right) \\
& P_{2}(z)=F^{2} \sin ^{2}\left(\frac{C}{F} z\right) \\
& F=\left[1+\frac{\left(\beta_{1}-\beta_{2}\right)^{2}}{4 C^{2}}\right]^{-1 / 2}
\end{aligned}
$$

Where $z$ is the distance along the coupling region, $C$ is the coupling coefficient. $\beta_{1}$ and $\beta_{2}$ are the propagation constants of the fundamental mode in the SMF and a certain high-order mode in the FMF, respectively. Supposing $\beta_{2}$ is the propagation constant of the LP11 mode in the TMF, when $\beta_{1}$ is equal to $\beta_{2}$, the phase mismatch $\Delta=\beta_{1}-\beta_{2}$ is equal to 0 , indicating that the fundamental mode (LP01) in the SMF and the high-order mode (LP11) in the TMF meet the phase matching condition. Then, Eqs. (1) and (2) can be simplified as: $P_{1}=\cos ^{2}(C z)$ and $P_{2}=\sin ^{2}(C z)$, indicating a complete periodic power exchange between the LP01 mode and the LP11 mode in the lossless case.

According to above theoretical analysis, it can be seen that as to achieve mode conversion from the LP01 mode to the LP11 mode, the phase matching condition must be satisfied: $\beta_{1}=\beta_{2}$. Furthermore the propagation constant $\beta$ can be calculated as: $\beta=n_{e f f} k_{0}$, where $k_{0}$ is the propagation constant in vacuum, $n_{\text {eff }}$ is the mode effective index. The phase matching condition can be converted to: $n_{\text {eff } 1}=n_{\text {eff } 2}$. Since the effective index of the mode varies with the diameter of the fiber, it is necessary to find the optimum fiber diameters in order to meet the phase matching condition. We calculated the mode effective index versus different fiber diameters by finite element method (FEM) as shown in Fig. 2. When the cladding diameter of the SMF is less than $6 \mu \mathrm{m}$ and the cladding diameter of the TMF is less than $9 \mu \mathrm{m}$, the effective index curve slope of the LP01 mode (in the SMF) equals to that of the LP11 mode (in the TMF). The best diameter ratio of the SMF as compared to the TMF is about 0.64 , so the cladding diameter of the SMF should be pre-tapered to $80 \mu \mathrm{m}$.



Fig. 2. The mode effective index of the LP01 mode (in the SMF) and the LP11 mode (in the TMF) versus different fiber cladding diameters at the wavelength of $1064 \mathrm{~nm}$

Then, modes' propagation in the MSC was calculated by beam propagation method (BPM) to confirm the phase matching condition. As shown in Fig. 3(a), the cladding diameters of SMF and TMF optical fibers are $5 \mu \mathrm{m}$ and $7.8 \mu \mathrm{m}$, respectively (the diameter ratio is about 0.64 ). Fig. 3(b) shows the power exchange in the coupling region when LP01 mode in the SMF is converted to LP11 mode in the TMF. The simulation results show that the mode powers are periodically exchanged in the coupling region of the MSC. Figs. 3(c)-(f) show the mode intensity distributions along the coupling region when LP01 mode converts into the LP11 mode in one period. (a)

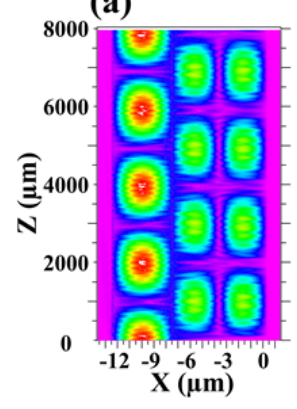

(b)

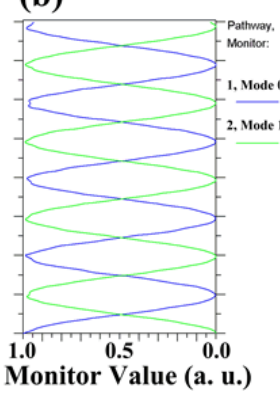

\section{(c)}

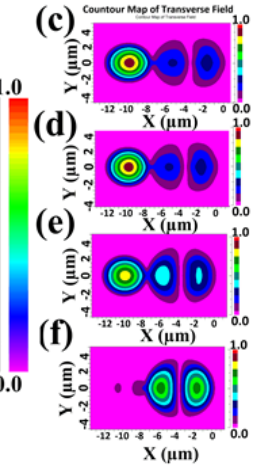

Fig. 3. Simulation results of: (a) Mode intensity distribution in the SMF and the TMF; (b) Power exchange in the coupling region when LP01 mode (in the SMF) converts to LP11 mode (in the TMF); (c)-(f) Evolutions of the mode intensity distributions in the coupling region when LP01 mode converts to LP11 mode in one period.

Because the modal effective refractive index varies with the shape of the fiber, we use weak fusion technique for maintaining the geometry of the SMF and the TMF in order to meet the phase matching condition and improve the mode purity [21], [23]. The advantages of using weakly fused 
technology are: weakly fused technology can maintain the geometry of both fibers (the coupling region of the coupler can be treated as two touching circular cylinders) and achieve accurate refractive index matching for phase matched mode conversion from LP01 mode (in the SMF) to LP11 mode (in the TMF). According to the above simulation results, in order to realize the mode conversion from LP01 mode to LP11 mode, SMF should be first pre-tapered from $125 \mu \mathrm{m}$ to $80 \mu \mathrm{m}$ by the flame modification method [24]. Then, the TMF was aligned with the pre-tapered SMF and they are fused together. When the SMF and the TMF are aligned, they are not twisted but placed in parallel in order to precisely control the mode conversion from LP01 mode (in the SMF) to LP11 mode (in the TMF). During the pulling process, a laser source with a wavelength near $1.0 \mu \mathrm{m}$ is launched into the SMF input port. Meanwhile, optical powers of the two output ports are monitored. Here we fabricated a MSC with a coupling efficiency of $10 \%$ (SMF: $90 \%$, TMF: $10 \%$ ) for the pulsed fiber laser described below. The mode intensity distributions at the TMF output port of the fabricated MSC are detected by a CCD detector (CinCam IR) near the wavelength of $1.0 \mu \mathrm{m}$, as shown in Fig. 4. Fig. 4(a) shows the mode intensity distribution of the two-lobed LP11 mode at the TMF output port. Fig. 4(b) shows the cross-sectional intensity distributions of the two-lobed LP11 mode. It can be seen that the intensity distributions in two lobes of the LP11 mode are symmetrical and the intensity of the dip between the two lobes is zero, thus the LP11 mode purity is very high, which is measured to be $>96 \%$ by the tight bend approach [25]. Also, the mode intensity distributions at the TMF output port with different wavelengths near $1.0 \mu \mathrm{m}$ are measured as in Figs. 4(c)-4(f). The loss of the MSC is measured to be about $0.5 \mathrm{~dB}$.

(a)



(b)
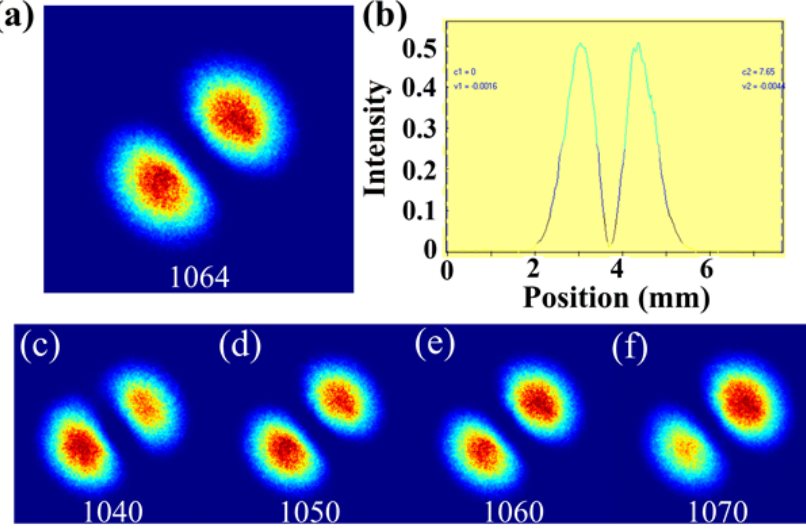

Fig. 4. CCD images of: (a) The measured mode intensity distribution at the TMF output port of the MSC; (b) The cross-sectional intensity distribution of the LP11 mode; (c)-(f) Mode intensity distributions of the LP11 mode excited in the TMF at different launching wavelengths.

The transmission spectra at the output of the SMF port and the TMF are measured by an optical spectrum analyzer (OSA, YOKOGAWA, AQ6370C), with an amplified spontaneous emission (ASE) source connected to the SMF input port. As show in Fig. 5(a), the transmission spectrum of the TMF output has weak interference phenomenon. The reason for this may be that a very small amount of LP01 in the output port of the TMF, due to mismatching in the coupler parameters resulted from some variation in the taper geometry, especially in the transition region of the coupler [21]. Fig. 5(b) shows the packaged MSC which has high environmental immunity and stability.

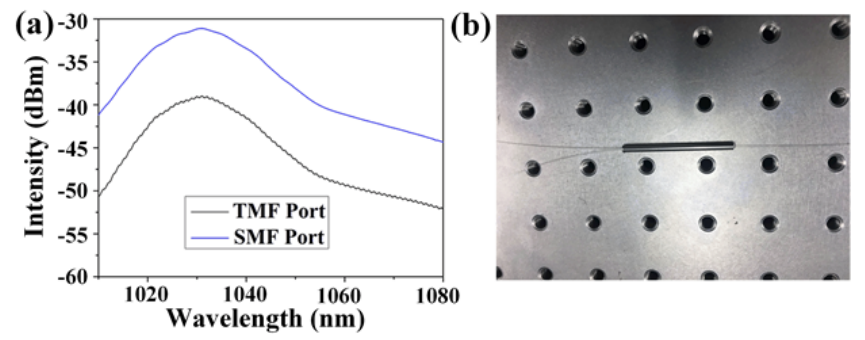

Fig. 5. Experimentally measured: (a) Transmission spectra at the two output ports of the MSC; (b) Image of the packaged MSC.

The experimental setup of the pulsed CVB fiber laser is illustrated in Fig. 6, which consists of a $974 \mathrm{~nm}$ laser diode (LD), a 980/1064 nm wavelength division multiplexer (WDM), a section of ytterbium-doped-fiber (YDF, Nufern, SM-YSF-HI), a circulator, a SESAM (Semiconductor Saturable Absorber Mirror, Batop), a coupler, three polarization controllers (PCs) and a MSC (90:10). The total cavity length is about $11 \mathrm{~m}$. The MSC acts as the transverse mode converter and mode splitter. The mode-locking mechanism is achieved due to the saturable absorption effect of the SESAM, which is sandwiched between two fiber connectors. The intensity absorption and absorber relaxation time of the SESAM are $18 \%$ and $500 \mathrm{fs}$, respectively. When the light is reflected by the SESAM into the MSC, the LP01 mode is converted into the LP11 mode and detected by CCD after a collimator. The spectral and temporal properties of the laser are measured at output 1 , by the OSA and an oscilloscope (OSC, SDA 6000A). The output beam profiles are captured by the CCD camera placed at output 2 through a fiber collimator.

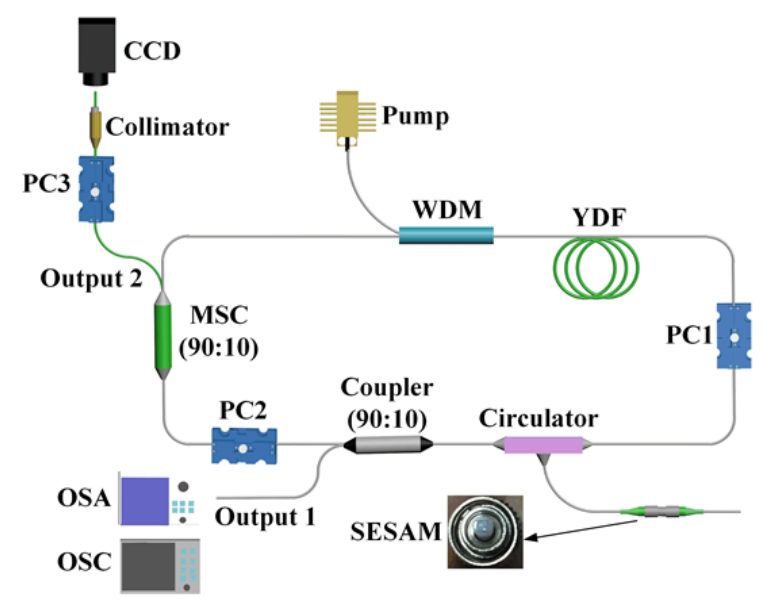

Fig. 6. Experimental setup and monitoring system of the pulsed CVB fiber laser. 


\section{MODE-LOCKED CVB FibER LASER AND RESUlTS}

The mode-locked laser operation with stable pulse trains can be easily obtained by adjusting the $\mathrm{PC} 1$ in the cavity, when pump power is above the mode-locking threshold value. The mode-locked laser spectrum with a pump power of $150 \mathrm{~mW}$ at output 1 is measured as Fig. 7(a). Since the dispersion in the laser cavity is all-normal-dispersion everywhere, the optical spectrum of the mode-locked pulse exhibits steeping edges, which is a typical output spectrum of an all-normal-dispersion fiber laser [26], [27]. The center wavelength of the laser is $1042.3 \mathrm{~nm}$ and the $3-\mathrm{dB}$ spectral width is measured to be 1.5 $\mathrm{nm}$. The signal-to-background ratio is about $33 \mathrm{~dB}$. The corresponding laser pulse trains are shown in Fig. 7(b), with $53.7 \mathrm{~ns}$ interval between two adjacent pulses, the repetition rate is calculated to be $18.6 \mathrm{MHz}$
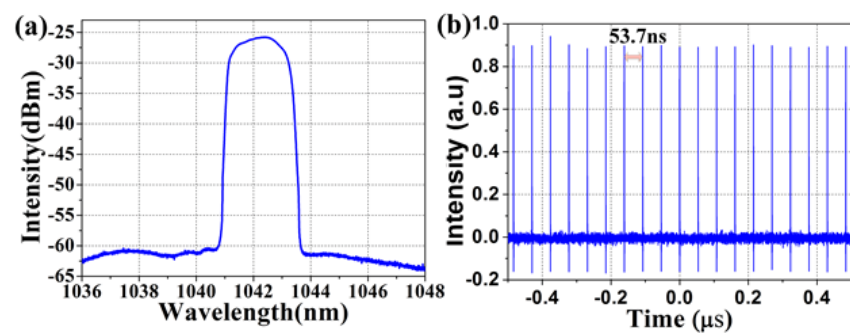

Fig. 7. Mode-locked laser output: (a) Mode locked laser spectrum with a pump power of $150 \mathrm{~mW}$; (b) Mode-locked pulse trains.

The RF spectrum of the laser is depicted in Fig. 8(a). The repetition rate is $18.6 \mathrm{MHz}$, which is in agreement with the calculated value according to cavity length. The electrical signal to noise ratio (SNR) measured with a $100 \mathrm{~Hz}$ resolution bandwidth (RBW) and a $0.6 \mathrm{MHz}$ frequency span is higher than $50 \mathrm{~dB}$. The inset of Fig. 8(a) is the RF spectrum without spectral modulation in a wide span range (up to $1 \mathrm{GHz}$ ), highlighting the low-amplitude fluctuation of the laser and indicating good stability of the mode-locked laser operation. Fig. 8(b) shows the output laser power versus the pump power (output 1, blue curve and output 2, black curve), the slope efficiency of output 1 (SMF output port) is about $6 \%$ and the slope efficiency of output 2 (TMF output port) is about $3.6 \%$.


Fig. 8. Experimental results: (a) RF spectrum of the mode-locked laser output measured with $0.6 \mathrm{MHz}$ frequency span and $100 \mathrm{~Hz}$ RBW. Inset: spectrum in $1 \mathrm{GHz}$ span; (b) Mode locked laser output power versus pump power (Black curve: output1-pump power, Blue curve: output2-pump power)

The MSC functions as a mode converter and splitter, when light passes through the MSC, the LP01 mode is converted into the LP11 mode and output from the TMF fiber port, which is connected to a collimator and detected by the CCD. The doughnut-shaped intensity profile of the radially and azimuthally polarized vector beams are obtained at the laser output 2 through adjustment of PC2 and PC3 as shown in Figs. 9(a) -9 (f). The purity of the radially polarized beam and the azimuthally polarized beam are measured to be $95.6 \%$ and $95.3 \%$, respectively [13], [19]. The radially and azimuthally polarized vector beams are confirmed by recording the intensity distributions by rotating a liner polarizer inserted between the collimator and the CCD camera. The mode intensity distributions of the radially and azimuthally polarized beams after passing a linear polarizer at different orientations are shown in Figs. 9(b)-9(e) and 9(g)-9(j), respectively.

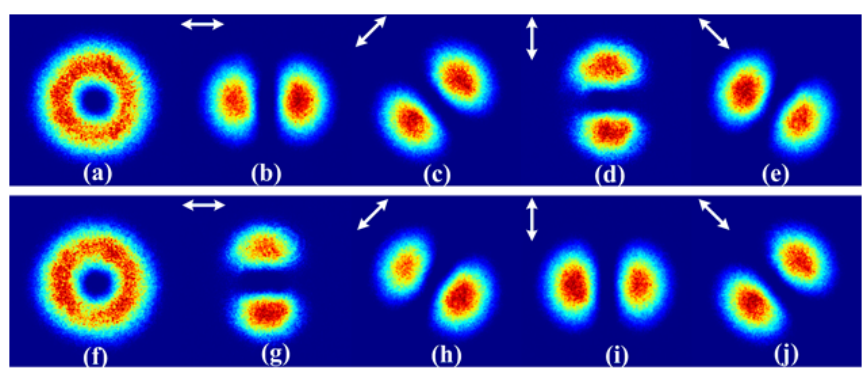

Fig. 9. Mode intensity distributions of: (a) Radially polarization beam and (f) Azimuthally polarization beam without a polarizer; (b)-(e): Radially polarization beam after passing a linear polarizer; (g)-(j): Azimuthally polarization beam after passing a linear polarizer. Arrow indicates the orientation of the linear polarizer.

\section{Conclusions}

In summary, for the first time to the best of our knowledge, we present a pulsed ytterbium-doped fiber laser generating CVBs with high efficiency and high mode purity. A MSC made by the weakly fused technology is used as the transverse mode converter and splitter. The MSC can achieve LP11 mode with a high purity of $>96 \%$ at the wavelength band of $1.0 \mu \mathrm{m}$ with a low loss of about $0.5 \mathrm{~dB}$. The mode-locked CVB pulses have a repetition rate of $18.58 \mathrm{MHz}$ with output power of $\mathrm{mW}$-level. The purities of the radially polarized beam and the azimuthally polarized vector beams are measured to be $95.6 \%$ and $95.3 \%$, respectively. This simple and novel CVB all-fiber laser may find applications in many areas such as optical tweezers, optical imaging, and mode-division multiplexed systems.

\section{REFERENCES}

[1] Q. Zhan, "Cylindrical vector beams: from mathematical concepts to applications," Adv. Opt. Photon., vol. 1, no. 1, pp. 1-57, Jan. 2009.

[2] R. Dorn, S. Quabis and G. Leuchs, "Sharper focus for a radially polarized light beam,” Phys. Rev. Lett., vol. 91, no. 23, pp. 233901-1-4, Dec. 2003.

[3] H. Kavauchi, K. Yonezawa, Y. Kozawa, and S. Sato, "Calculation of optical trapping forces on a dielectric sphere in the ray optics regime produced by a radially polarized laser beam," Opt. Lett., vol. 32, no. 13, pp. 1839-1841, Jul. 2007.

[4] R. S. R. Ribeiro, O. Soppera, A. G. Oliva, A. Guerreiro and P. A. Jorge, "New trends on optical fiber tweezers," J. Lightwave Technol., vol. 33, no. 16, pp. 3394-3405, Aug. 2015. 
[5] M. Meier, V. Romano, and T. Feurer, "Material processing with pulsed radially and azimuthally polarized laser radiation," Appl. Phys., A Mater. Sci. Process., vol. 86, no. 3, pp. 329-334, Mar. 2006.

[6] L. Novotny, M. R. Beversluis, K. S. Youngworth, and T. G. Brown, "Longitudinal field modes probed by single molecules," Phys. Rev. Lett., vol. 86, no. 23, pp. 5251-5254, Jun. 2001.

[7] A. Bouhelier, F. Ignatovich, and A. Bruyant, "Surface plasmon interference excited by tightly focused laser beams, " Opt. Lett., vol. 32, no. 17, pp. 2535-2537, Sep. 2007.

[8] J. L. Li, K. Ueda, and M. Musha, "Generation of radially polarized mode in $\mathrm{Yb}$ fiber laser by using a dual conical prism,” Opt. Lett., vol. 31, no. 20, pp. 2969-2971, Oct. 2006.

[9] M. P. Thirugnanasambandam, Y. Senatsky, and K.Ueda. "Generation of radially and azimuthally polarized beams in Yb:YAG laser with intra-cavity lens and birefringent crystal,” Opt. Express, vol. 19, no. 3, pp. 1905-1914, Jan. 2011.

[10] S. Ngcobo, I. Litvin, and L. Burger, "A digital laser for on-demand laser modes," Nat. Commun., vol. 44, no. 2289, Aug. 2013.

[11] D. Lin, K. Xia, and J. Li, "Efficient, high-power, and radially polarized fiber laser," Opt. Lett., vol. 35, no. 13, pp. 2290-2292, Jul. 2010.

[12] H. Wan, H. Li, and C. Wang, "An Injection-Locked Single-Longitudinal-Mode Fiber Ring Laser with Cylindrical Vector Beam Emission," IEEE Photon. J., vol. 9 , no. 1, pp. 1-8, Feb. 2017.

[13] B. Sun, A. Wang, and L. Xu, "Low-threshold single-wavelength all-fiber laser generating cylindrical vector beams using a few-mode fiber Bragg grating," Opt. Lett., vol. 37, no. 4, pp. 464-466, Feb. 2012.

[14] Y. Zhou, A. Wang, and C. Gu, "Actively mode-locked all fiber laser with cylindrical vector beam output," Opt. Lett., vol. 41, no. 3, pp. 548-550, Feb. 2016.

[15] Y. Zhou, J. Lin, and X. Zhang, "Self-starting passively mode-locked all fiber laser based on carbon nanotubes with radially polarized emission," Photon. Research, vol. 4, no. 6, pp. 327-330, Dec. 2016.

[16] B. Sun, A. Wang, and C. Gu, "Mode-locked all-fiber laser producing radially polarized rectangular pulses," Opt. Lett., vol. 40, no. 8, pp. 1691-1694, Apr. 2015.

[17] K. Yan, J. Lin, and Y. Zhou, " $\mathrm{Bi}_{2} \mathrm{Te}_{3}$ based passively Q-switched fiber laser with cylindrical vector beam emission," Appl. Opt., vol. 55, no, 11, pp. 3026-3029, Apr. 2016.

[18] F. Wang, F. Shi and T. Wang, "Method of generating femtosecond cylindrical vector beams using broadband mode converter," IEEE Photon. Technol. Lett., vol. 29, no. 9, pp. 747-750, May 2017.

[19] H. Wan, J. Wang, and L. Zhang, "High efficiency mode-locked, cylindrical vector beam fiber laser based on a mode selective coupler," Opt. Express, vol. 25, no. 10, pp. 11444-111451, May 2017.

[20] Z. Zhang, Y. Cai and J. Wang, "Switchable dual-wavelength cylindrical vector beam generation from a passively mode-locked fiber laser based on carbon nanotubes," IEEE J. Sel. Top. Quantum Electron., vol. 24, no. 3, pp, 1-6, May. 2018.

[21] R. Ismaeel, T. Lee, and B. Oduro, "All-fiber fused directional coupler for highly efficient spatial mode conversion,” Opt. Express, vol. 22, no. 10, pp. 11610-11619, May 2014.

[22] Y. L. Xiao, Y. G. Liu, W. Zhi, Z. Wang, and X. Q. Liu, "Design and experimental study of mode selective all-fiber fused mode coupler based on few mode fiber," Acta. Phys. Sinica., vol. 64, no. 20, Oct. 2015.

[23] G. Pelegrina-Bonilla, K. Hausmann, and H. Sayinc. "Analysis of the modal evolution in fused-type mode-selective fiber couplers," Opt. Express, vol. 23, no. 18, pp. 22977-22990, Sep. 2015.

[24] G. Brambilla, "Optical fibre nanowires and microwires: A review," J. of Opt., vol. 12, no, 4, Apr. 2010.

[25] K. Y. Song, I. K. Hwang, and S. H Yun, "High performance fused-type mode-selective coupler using elliptical core two-mode fiber at $1550 \mathrm{~nm}$," IEEE Photon. Technol. Lett., vol. 14, no. 4, pp. 501-503, Apr. 2002.

[26] A. Chong, W. H. Renninger and F. W. Wise, "Environmentally-stable all-normal-dispersion femtosecond fiber laser," Opt. Lett., vol. 33, no. 10, pp. 1071-1073, May. 2008.
[27] A. Chong, W. H. Renninger and F. W. Wise. "All-normal-dispersion femtosecond fiber laser with pulse energy above 20 nJ," Opt. Lett., vol. 32, no. 16, pp. 2408-2410, Aug. 2007. 